\title{
Measurement of gastrointestinal transmural electric potential difference in man
}

MICHAEL G. GEALL, CHARLES F. CODE, DONALD C. MCILRATH, AND W. H. J. SUMMERSKILL

From the Gastroenterology Unit, and the Section of Physiology and of Surgery, Mayo Clinic and Mayo Foundation, Rochester, Minnesota, USA

SUMMARY Measurement, in man, of the electric potential difference between venous blood and the mucosal surface of the gastrointestinal tract gave identical values to the potential difference between mucosa and serosa. Various parts of the peritoneum were equipotential with venous blood. By contrast, skin-enteric potential difference varied with time and among different subjects because of a potential difference between skin and blood that is unpredictably reduced by skin injury. The results with electrolyte bridges of $\mathrm{KCl}$ in agar or of flowing $\mathrm{KCl}$ were identical.

Electric potential differences across the wall of the gastrointestinal tract were recognized nearly 150 years ago (Donné, 1834). Ussing, Kruhøffer, Thaysen, and Thorn (1960) originally emphasized the importance of potential differences in intestinal electrolyte transport, and the association of gastric mucosal damage with reduced transmucosal potential differences has suggested (Davenport, Warner, and Code, 1964) that measurements of potential difference in the stomach may be an index of mucosal integrity.

Steady potential differences have been measured directly in animal preparations both in vivo and in vitro (Barry, 1967; Durbin, 1967). When the reference electrode was placed in the blood, the potential difference was the same as that between serosa and mucosa (Cooperstein and Brockman, 1959; Edmonds, 1967), and placing the electrode intravenously has been used routinely by some (Davenport et al, 1964). In man, study of potential difference has been hindered by the absence of a validated technique whereby absolute measurements can be obtained. The customary site of the indifferent electrode on abraded or unabraded skin (Adair and Goodman, 1936; Andersson and Grossman, 1965; Fordtran, Rector, and Carter, 1968; Helm,

${ }^{1}$ This investigation was supported in part by research grant AM-6908 from the National Institutes of Health, Public Health Service.
Schlegel, Code, and Summerskill, 1965; Ingram and Richards, 1953; Meckeler and Ingelfinger, 1967; Morton, 1954; Rice and Ross, 1947; Rovelstad, 1956) yielded variable results, which did not accord with direct readings (Morton, 1954). More recently, Andersson and Grossman (1965) demonstrated that a large and variable potential difference originated from unabraded skin, and this accounted for the differences between blood-enteric and skin-enteric potential differences. The finding of Soergel, Whalen, Geenen, and Gustke (1966) that the potential difference between an unspecified region of peritoneum and peripheral blood was less than $1 \mathrm{mv}$ in four subjects undergoing peritoneal dialysis also supported the use of peripheral blood as equipotential to serosa or the peritoneal cavity in determining transmural potential difference in man.

Because use of peripheral blood for the site of a reference electrode has not been directly confirmed in man in determining transmural potential differences, measurements were made at operation between mucosa and serosa and compared with those between mucosa and peripheral blood. Measurements were also made in conscious healthy subjects of the potential difference between peripheral blood and gastrointestinal mucosa and between skin and gastrointestinal mucosa. It was found that use of peripheral blood 
yielded accurate results for measurement of transmural potential difference, whereas no acceptable correction factor was identified for skin-enteric determination of the potential difference.

Renewal of the liquid junction between the contents of the electrolyte bridge and the gastrointestinal secretions is desirable in an attempt to eliminate junction potentials. The use of flowing $\mathrm{KCl}$, which contains fast-moving ions of equal mobilities, reduces this tendency (Hollander, 1944), but a bridge of $\mathrm{KCl}$ in agar is safer and more convenient. To detect possible artifacts due to junction potentials resulting from agar, a comparison of the two methods was also made.

\section{Material and Methods}

Direct measurements of the electric potential difference between the gastrointestinal mucosa and the serosa and between the mucosa and the peripheral blood were made on grossly normal regions of the gastrointestinal tract in 11 patients undergoing abdominal operations. Other studies were carried out on healthy, fasting male volunteers, whose ages ranged between 23 and 36 years.

The technique of measuring potential difference is identical to that used by Davenport et al (1964) and by Andersson and Grossman (1965). The electrolyte bridges of the gastrointestinal electrode consisted of two polyethylene tubes (ID $0.17 \mathrm{~cm}$ ). One contained saturated $\mathrm{KCl}$ in $3 \%$ agar, whereas saturated $\mathrm{KCl}$ was used for the other. The intravenous electrolyte bridge consisted of a polyethylene tube (ID $0.08 \mathrm{~cm}$ ) containing saturated $\mathrm{KCl}$ in $3 \%$ agar (clamped at the ends during sterilization by gas beforehand), and placed in the vein through a 15-gauge needle. Each electrolyte bridge rested in a beaker containing saturated $\mathrm{KCl}$ solution and two balanced calomel half-cells (Beckman fibre-junction type) connected to a $p \mathrm{H}$ meter (Beckman Expandomatic), which acted as a high-impedance directcurrent millivoltmeter. The potential difference was recorded continuously by a visual recorder (Honeywell Visicorder) calibrated immediately before and checked immediately after each study.

The intestinal electrolyte bridge was passed through the nose into the gastrointestinal tract and, at laparotomy, the potential difference between this and an electrolyte bridge placed on the serosa opposite the intraluminal electrode was measured. Then, with the mucosal bridge in the same place, the potential difference between mucosa and a bridge in a peripheral vein was measured. When measurements of skin-enteric, skin-blood, and blood-enteric potential difference were made in healthy volunteers, the intestinal electrolyte bridge was positioned fluoroscopically and the potential difference was continuously recorded. All bridges used were of the agar- $\mathrm{KCl}$ type except for the comparison of the two electrolyte bridges. Readings of skin-enteric potentials utilized the unabraded index finger immersed in saturated $\mathrm{KCl}$ solution as the point of reference. Potential difference was measured continuously for six minutes in all instances after steady conditions became established.

The comparison between the potential difference recorded by flowing $\mathrm{KCl}$ and that by agar$\mathrm{KCl}$ bridges was made after the bridges had been exposed to digestive action in the gastrointestinal tract for at least three hours in the stomach (after maximal betazole stimulation) or at least for 12 hours in the small intestine.

\section{Results}

Comparison of mucosa-to-serosa and mucosa-toblood potential differences in the same patients at laparotomy showed a highly significant correlation ( $r=0.99)$ (Fig. 1). In six of these patients, no measurable difference in potential difference existed between peripheral blood and the serosa of the colon, small intestine, stomach, or parietal peritoneum.

In healthy persons, the difference between skinmucosal and blood-mucosal potential difference approximated the potential difference between skin and blood (Fig. 2). Both the skin and gastrointestinal mucosa were negative with respect to blood. Skin-mucosal potential difference did not become stable until about five minutes and varied greatly among different subjects, whereas bloodmucosal potential difference did not change significantly (Fig. 2). Skin-blood potential difference was reduced by skin injury and was lower if recorded from regions of the body other than the fingers (Table). No difference $(r=1.00)$ in potential difference was found when flowing $\mathrm{KCl}$ and agar- $\mathrm{KCl}$ electrolyte bridges were used (Fig. 3).

\begin{tabular}{llll}
\hline & \multicolumn{3}{l}{ Potential Difference $(m v)$} \\
\cline { 2 - 4 } Site & Mean & SD & SEM \\
\hline Unabraded finger & 34.8 & 10.0 & 0.97 \\
Abraded finger & 15.9 & 2.1 & 0.53 \\
Arm & 17.4 & 3.2 & 0.66 \\
Abraded arm & 7.6 & 0.3 & 0.11 \\
Leg & 22.9 & 7.1 & 1.47 \\
\hline
\end{tabular}

Table Potential differences between injured or uninjured skin and peripheral blood in three normal subjects

\section{Discussion}

These results in man accord with animal observations that the potential difference between mucosa and serosa is the same as between mucosa and peripheral venous blood (Cooperstein and Brockman, 1959; Edmonds, 1967). Presumably 


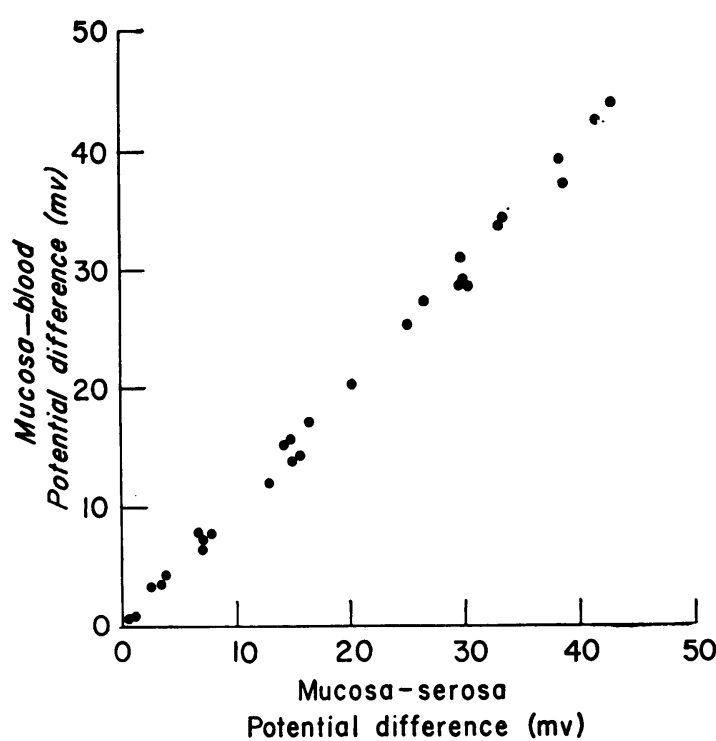

Fig. 1 Comparison of mucosa-serosal potential difference with mucosal-blood potential difference at laparotomy: mucosal measurement from stomach (13), small intestine (9), or colon (6).

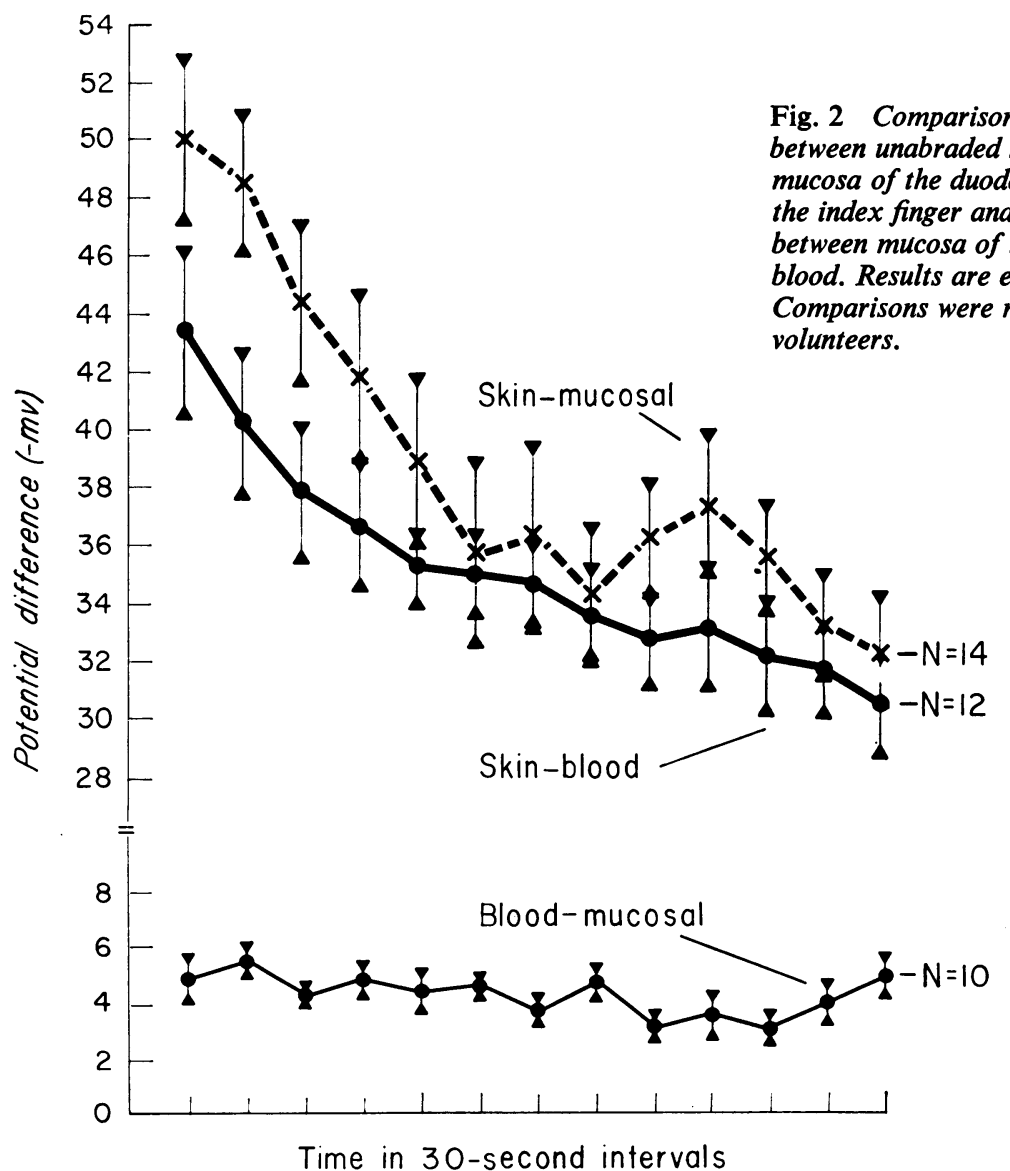

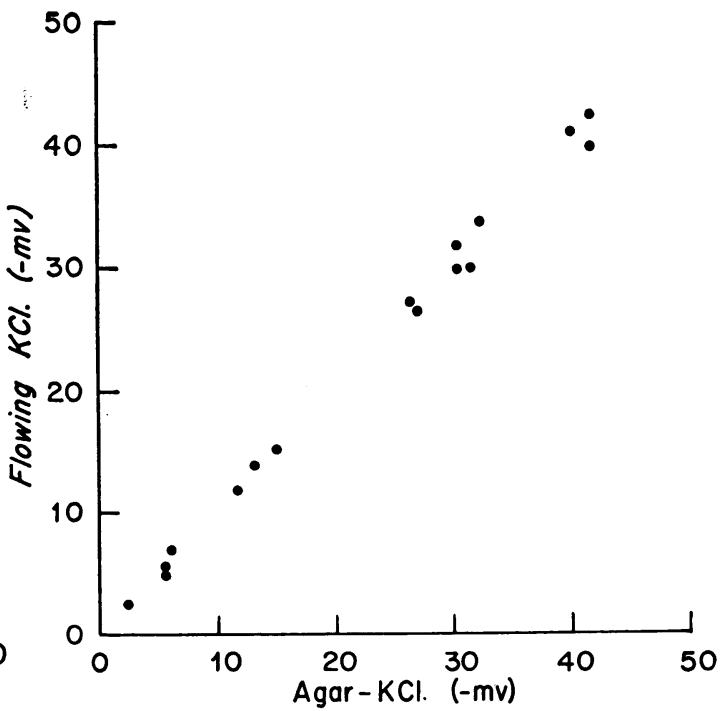

Fig. 3 Comparison of potential difference values obtained from identical regions of the gastrointestinal tract using flowing $\mathrm{KCl}$ and agar-KCl electrolyte bridges $(N=16)$. 
the blood acts as a highly effective electrolyte bridge, bringing the reference electrode into electric contact with the cell of origin of the potential difference. By contrast, the potential difference between the skin and gut was large, varied with time, and differed among persons; no correction factor can be applied. If the skin potential is abolished by injury, the potential difference should be equal to that in the peripheral blood. However, lesser degrees of skin injury would still leave a measurable potentialdifference, and, in order to establish that the skin potential had been abolished, the potential difference, between the abraded skin and the peripheral blood must be excluded.

Use of peripheral blood as reference allows investigation of transmural potential difference of the gastrointestinal tract in intact unanaesthetized man, and introduction of the intravenous electrolyte bridge is safe and simple. Readings with the flowing $\mathrm{KCl}$ bridge and the agar- $\mathrm{KCl}$ bridge were identical and no artifacts could be detected, although suspension of $\mathrm{KCl}$ in agar might theoretically encourage the formation of a sharper liquid junction and the appearance of junction potentials. These findings support the use of $\mathrm{KCl}$ in agar as an electrolyte bridge. Now that absolute measurements of transmural electric potential differences are possible in the gastrointestinal tract of unanaesthetized man, the technique can be applied to investigations of transport mechanisms and the pathophysiology of various disease states.

\section{References}

Adair, G. S., and Goodman, E. N. (1936). A note on the potential difference across the stomach membranes in human subjects, and a simple type of calomel electrode. J. Physiol. (Lond.), 87, 35-36P.

Andersson, S., and Grossman, M. I. (1965). Profile of $p \mathrm{H}$, pressure, and potential difference at gastroduodenal junction in man. Gastroenterology, 49, 364-371.

Barry, R. J. C. (1967). Electrical changes in relation to transport. Brit. med. Bull., 23, 266-269.

Cooperstein, I. L., and Brockman, S. K. (1959). The electrical potential difference generated by the large intestine: Its relation to electrolyte and water transfer. J. clin. Invest., 38, 435-442.

Davenport, H. W., Warner, H. A., and Code, C. F. (1964). Functional significance of gastric mucosal barrier to sodium. Gastroenterology, 47, 142-152.

Donné, A. (1834). Recherches sur quelques unes des propriétés chimiques des sécrétions, et sur les courants électriques qui existent dans les corps organisés. Ann. Chim. Phys., 57, 398-416.

Durbin, R. P. (1967). Electrical potential difference of the gastric mucosa. In Handbook of Physiology; Section 6: Alimentary Canal, edited by C. F. Code and W. Heidel, vol. 2, Secretion, pp. 879-888. American Physiological Society, Washington, D.C.

Edmonds, C. J. (1967). The gradient of electrical potential difference and of sodium and potassium of the gut contents along the caecum and colon of normal and sodiumdepleted rats. J. Physiol. (Lond.), 193, 571-588.

Fordtran, J. S., Rector, F. C. Jr., and Carter, N. W. (1968). The mechanisms of sodium absorption in the human small intest ine. J. clin. Invest., 47, 884-900.

Helm, W. J., Schlegel, J. F., Code, C. F., and Summerskill, W. H. J. (1965). Identification of the gastroesophageal mucosal junction by transmucosal potential in healthy subjects and patients with hiatal hernia. Gastroenterology, 48, 25-35.
Hollander, F. (1944). The measurement of electrical potentials in the stomach. Gastroenterology, 3, 319-322.

Ingram, P. W., and Richards, D. L. (1953). Observations on potential differences of the human stomach by a new electrode and measuring system. Gastroenterology, 25, 273-289.

Meckeler, K. J. H., and Ingelfinger, F. J. (1967). Correlation of electric surface potentials, intraluminal pressures, and nature of tissue in the gastroesophageal junction of man. Gastroenterology, 52, 966-971.

Morton, H. S. (1954). The potentialities of the electrogastrograph. Canad. Serv. med. J., 10, 233-243.

Rice, H. V., and Ross, R. T. (1947). Factors affecting the electrical potential of the gastric mucosa. Amer. J. Physiol., 149, 77-94.

Rovelstad, R. A. (1956). Continuously recorded in situ $p \mathrm{H}$ of gastric and duodenal contents in patients with and without duodenal ulcers. Gastroenterology, 31, 530-537.

Soergel, K. H., Whalen, G. E., Geenen, J. E., and Gustke, R. F (1966). Potential difference of the intact human intestine during active and passive transport. (Abstr.) Proc. Cent. Soc. clin. Res., 39, 75.

Ussing, H. H., Kruhoffer, P., Taysen, J. H., and Thorn, N. A. (1960). The alkali metal ions in biology, In Handbuch der experimentellen Pharmakologie (Suppl.), edited by A. Heffter and W. Heubner, vol. 13, p. 59. Springer, Berlin. 